\title{
Axillary Reconstruction for Hidradenitis Suppurativa with an Inner-Arm Transposition Flap Creating a Brachioplasty Effect
}

\author{
Daniel L. Ching ${ }^{1}$, Maleeha Mughal ${ }^{2}$, Athanasios Papas ${ }^{2}$, Mark Soldin ${ }^{2,3}$ \\ ${ }^{1}$ Department of Anatomical Pathology, Queen Elizabeth II Medical Centre, Perth, Australia; ${ }^{2}$ Department of Plastic and Reconstructive \\ Surgery, St George's Healthcare NHS Trust, London; ${ }^{3}$ Department of Plastic and Reconstructive Surgery, Kingston Healthcare NHS Trust, \\ London, UK
}

Background Hidradenitis suppurativa (HS) is a chronic skin condition that can affect any area with apocrine sweat glands and has the potential to involve multiple sites concurrently. Commonly affected sites include the axilla, groin, perineum and perianal areas. In this study we performed a literature review on the surgical methods for HS and describe an innovative technique for reconstructing axilla HS using an inner-arm transposition flap.

Methods We reviewed all cases ( 5 cases from 4 patients) of transposition flap reconstruction performed by the senior author at a single London tertiary hospital from 2008-2013. Patient related outcome measures were collected using the Derriford appearance scale (DAS 24) and a study specific questionnaire.

Results All patients were satisfied with their final result. One out of five cases had a complication but did not result in flap failure. There is no disease recurrence to date. DAS 24 scores collected demonstrated acceptable postoperative distress that did not deviate far from the norm tables while study specific questionnaire reveal desirable outcomes.

Conclusions We have managed to achieve our aim through the use of the innovative innerarm transposition flap. Our study hopes to provide an additional technique for axillary reconstruction. This technique offers the effective concealment of scars with the benefit of tightening of the arm tissue producing 'brachioplasty like' effects. All things considered it would be reasonable to conclude the innovative flap technique is a reliable, effective, and simple method that results in multiple benefits.

Keywords Hidradenitis suppurativa / Axilla / Surgical flap
Correspondence: Daniel L. Ching Department of Anatomical Pathology, Queen Elizabeth II Medical Centre, Perth, Australia

E-mail: Danielching.lh@gmail.com

This article was presented 12th European Society of Plastic, Reconstructive and Aesthetic Surgery Congress, 8 July 2014, Edinburgh, UK.

No potential conflict of interest relevant to this article was reported.

Received: 9 Aug 2016 • Revised: 29 Oct 2016• Accepted: 14 Dec 2016

pISSN: 2234-6163・ elSSN: 2234-6171 • https://doi.org/10.5999/aps.2017.44.3.228・ Arch Plast Surg 2017;44:228-233

\section{INTRODUCTION}

Hidradenitis suppurativa (HS) is a chronic skin condition affecting the apocrine glands. The natural course of this disease leads to formation of abscesses, sinuses and fibrosis resulting in chronic wounds, which significantly impacts on the lives of the affected patients. The incidence of HS in the UK is estimated at $1: 600$, whereas worldwide prevalence is $1 \%$ of general population, with a female to male ratio of $2-5: 1[1,2]$.

Historically HS was thought to be a disease of the sweat glands 
based purely on Velpeau's description of anatomical distribution of the disease in the axilla and perineum. It was in 1922 that the disease was localized to the apocrine glands [3]. The histological features were initially described by Brunsting in 1939, with further histological studies confirming the disease to be a defect in the follicular epithelium [4].

HS can affect any area with apocrine sweat glands and has the potential to involve multiple sites concurrently. Commonly affected sites include the axilla, groin, perineum and perianal areas [5]. It is more common in the axilla for women and the perineal region in men [6]. Axillary HS is bilateral in $75 \%$ of cases [7]. The exact aetiology of HS still remains unclear; however, possible cited associations include cigarette smoking, obesity, diabetes mellitus, and Crohn's disease [8,9].

HS is a chronic debilitating condition that has widespread functional and psychosocial implications. Patients have difficulty with maintaining hygiene, are prone to recurrent infections and report a poor quality of life [5]. Disease severity can range from mild to life threatening and the Hurley's staging classification is a useful tool to guide management [10]. Some authors argue that in small, localised cases, antibiotics may have a role in disease control [11], however all conservative therapies do not prevent recurrence. The only definitive form of treatment is surgical excision $[12,13]$. Multiple methods of resection and wound closure have been described, but there is no consensus as to which one has the best outcome [13].

In this study we performed a literature review on the surgical methods for HS and describe an innovative method of reconstructing axillary HS using an inner-arm transposition flap resulting in the effects similar to that of a brachioplasty.

\section{METHODS}

\section{Data collection}

We reviewed all cases ( 5 cases in 4 patients) of inner-arm transposition flap reconstruction performed by the senior author at a single London teaching hospital from 2008-2013 (Table 1). The aim of the reconstructive procedure was to treat axillary HS and prevent recurrence, collectively achieving minimal scarring at the excised area and donor site. Patient related outcome was collected using the Derriford appearance scale (DAS 24) [14] and a study specific questionnaire.

Included in our study were all HS cases referred to the senior author that fulfilled the criteria of Hurley's stage 3 disease, recurrent/persistent cases unresponsive to conservative treatment, severe cases affecting a patient's quality of life and cases amenable to surgery. We excluded cases with mild to moderate disease severity including Hurley's stage 1 and stage 2 diseases, cases that were under control with conservative treatment and patients declining surgical intervention. All patients were followed up by the plastic surgery team, wound nurse and physiotherapist for up to 4 weeks after surgery, with further ongoing plastic surgery follow up for all patients every 6 months.

\section{Inner-arm transposition flap surgical method}

The procedure was carried out under general anaesthesia with the patient positioned supine and the arm abducted at 90 degrees. The first phase involves wide local excision (WLE) of areas with active disease including the affected underlying soft tissue. The extent of the WLE is determined clinically and entails 0.5 to $1 \mathrm{~cm}$ of healthy skin peripheral to the affected areas to ensure adequate excision. The flap required to fill this defect is then marked on the inner arm (Fig. 1A). The midline of the marked flap is centred over the bicipital groove. A Doppler is not required during this process. The incision lines are then infiltrated with a combination of a dilute vasoconstrictor (1:500,000 adrenaline) and local anaesthetic in normal saline. After excision of the axillary disease (the dissection extends deep to all hard nodules/sinuses into the normal soft axillary subcutaneous fatty tissue), haemostasis is achieved and the size of the wound re-evaluated (Fig. 1B, C).

The second phase was the resurfacing of the tissue defect with an inner-arm transposition flap. The flap is raised in the subfascial plane from distal to proximal (Fig. 1D). The dissection is straight forward but close to brachial neurovascular structures and needs to be done carefully. Perforators from the brachial artery are encountered during the dissection. If the movement of the flap is impeded by a perforator vessel, then the vessel is di-

\section{Table 1. Hidradenitis suppurativa cases performed by senior author over a 4 year perio}

\begin{tabular}{|llcllcccc|}
\hline Patient & Side & $\begin{array}{c}\text { Inpatient stay } \\
\text { (day) }\end{array}$ & \multicolumn{1}{c}{ Details } & Complications & $\begin{array}{c}\text { Follow-up } \\
\text { (mo) }\end{array}$ & Recurrence & $\begin{array}{c}\text { Patient } \\
\text { satisfaction }\end{array}$ \\
\hline 1 & Left & 2 & Scarred areas+1 cm margin & Flap edges debrided & 36 & No & Yes \\
2 & Right & 2 & Wide local excision & None & 20 & No & Yes \\
3 & Left & 1 & Wide local excision & None & 15 & No & Yes \\
& Right & 1 & Wide local excision & None & 12 & No & No & Yes \\
4 & Left & 1 & Wide local excision & None & & & Yes \\
\hline
\end{tabular}


Fig. 1. Inner-arm transposition flap surgical method demonstrated on a patient

(A) The area marked out and flap design. (B) Excision of affected area. (C) Excised defect. (D) Flap raised. (E) Flap inset and closure.
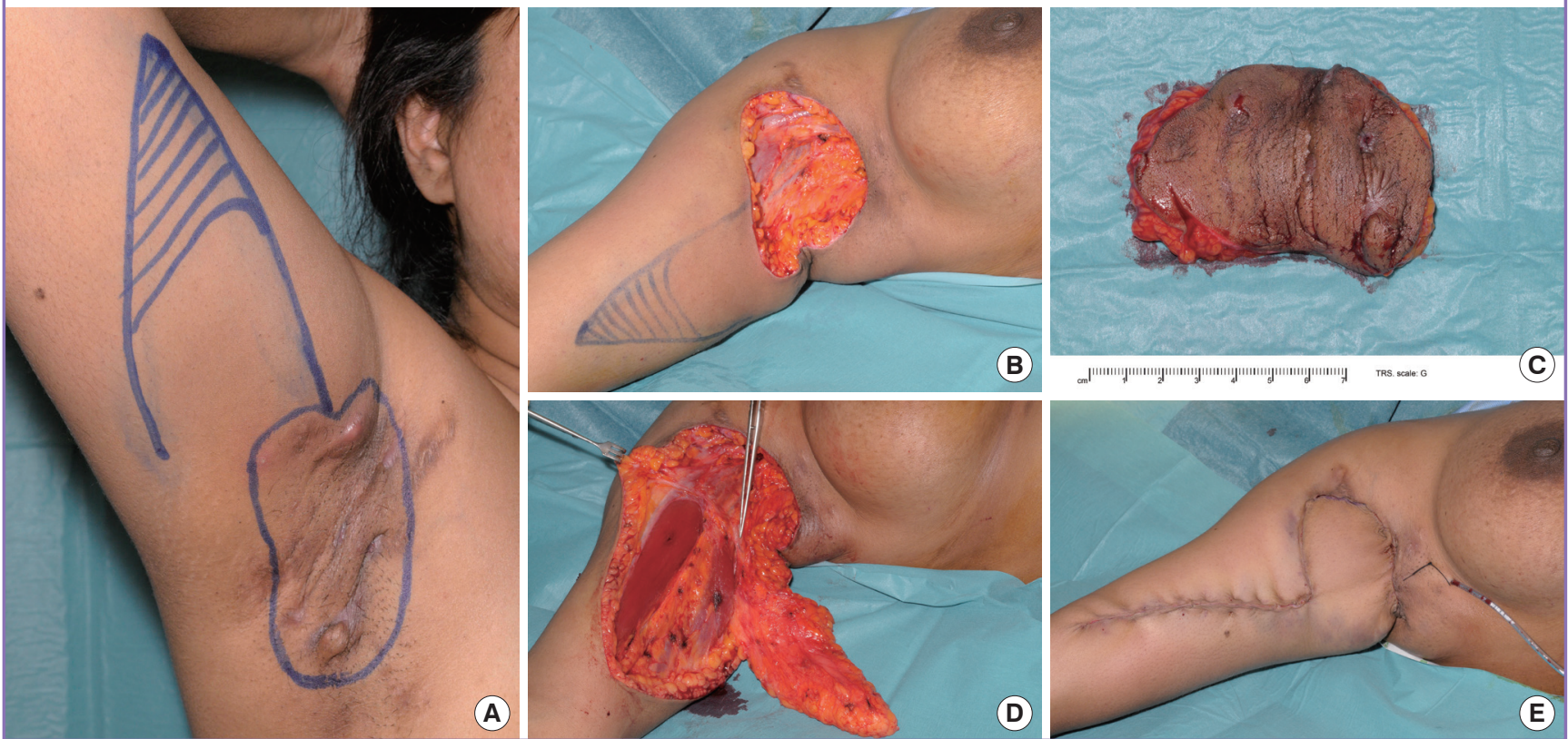

(A)
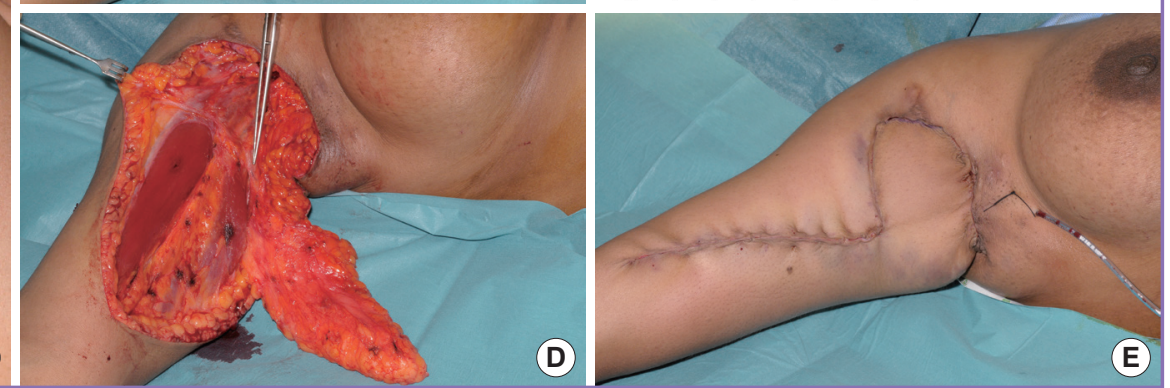

vided and the dissection continued proximally. When the flap is sufficiently mobilised to transpose into the defect the vascularity is reviewed.

In all cases there was a proximal brachial artery perforator that was preserved near the base of the flap. The flap length is trimmed to size checking tip vascularity and transposed into the axillary defect. The flap thickness usually matches the depth of the defect well. Once haemostasis is achieved, closure of both donor site and axillary wound is performed in two layers with 3-0 Monocryl and either 3-0 Nylon or staples to the skin (Fig. 1E). A small size 10 suction drain is placed prior to closure. The flap dimensions may need to be altered but this is unusual. Primary closure of the donor site completes the "brachioplasty like effect' with the scar extending down the inner arm and the donor site is closed as a linear scar without the need for skin graft. There is surrounding laxity within the axillary tissues and it is always possible to obtain primary wound closure of both donor site and axillary wound.

\section{RESULTS}

All four patients were female, with an average age of 34 (range, 24-49). The average follow up was 18 months (range, 8-36), and no recurrences were noted. Despite the severity of disease and extensive fibrosis at time of excision, we did not experience cases that required deeper than subcutaneous plane dissection.

One patient suffered a complication (necrosis of flap edges) requiring readmission and debridement 9 days postoperative. The patient was discharged 3 days later with subsequent follow up in clinic being uneventful. There were no other complications and there were no cases of complete flap failure. Patient 3 recognised the improved arm contour and requested symmetrisation. She later underwent bilateral reconstruction (Fig. 2). In cases of unilateral reconstruction, arm asymmetry was not reported to affect patient's perception of appearance. All patients were satisfied with their final result. There is no disease recurrence to date. In our small series, operating on a variety of larger and smaller circumference arms, no limitation of flap harvest or rotation was encountered.

The validated DAS 24 was used to assess postoperative satisfaction. Scores obtained were compared against the norm tables provided by the DAS 24 guide; high scores represent a distressed patient. Out of the four females, the two younger females aged 18-30 scored 56 (vs. norm 53.7), whereas the two older females aged 31-60 scored 46 (vs. norm 52.2). Scores collected demonstrate acceptable postoperative distress that did not deviate far from the norm tables.

We also used a study specific questionnaire consisting of 8 questions on a 6 point Likert Scale, ranging from strongly agree (SA), agree (A), neither agree nor disagree (NAND), disagree (D), strongly disagree (SD) and not applicable (N/A). Half of the patients strongly agreed that their quality of life improved and felt more confident after surgery; $75 \%$ of the patients strongly agreed when asked about satisfaction with end result. A 
Fig. 2. Patient 3 post reconstruction

(A) Right side reconstruction 8 months postoperative. (B) Left side reconstruction 15 months postoperative. (C) Left side reconstruction lateral view.
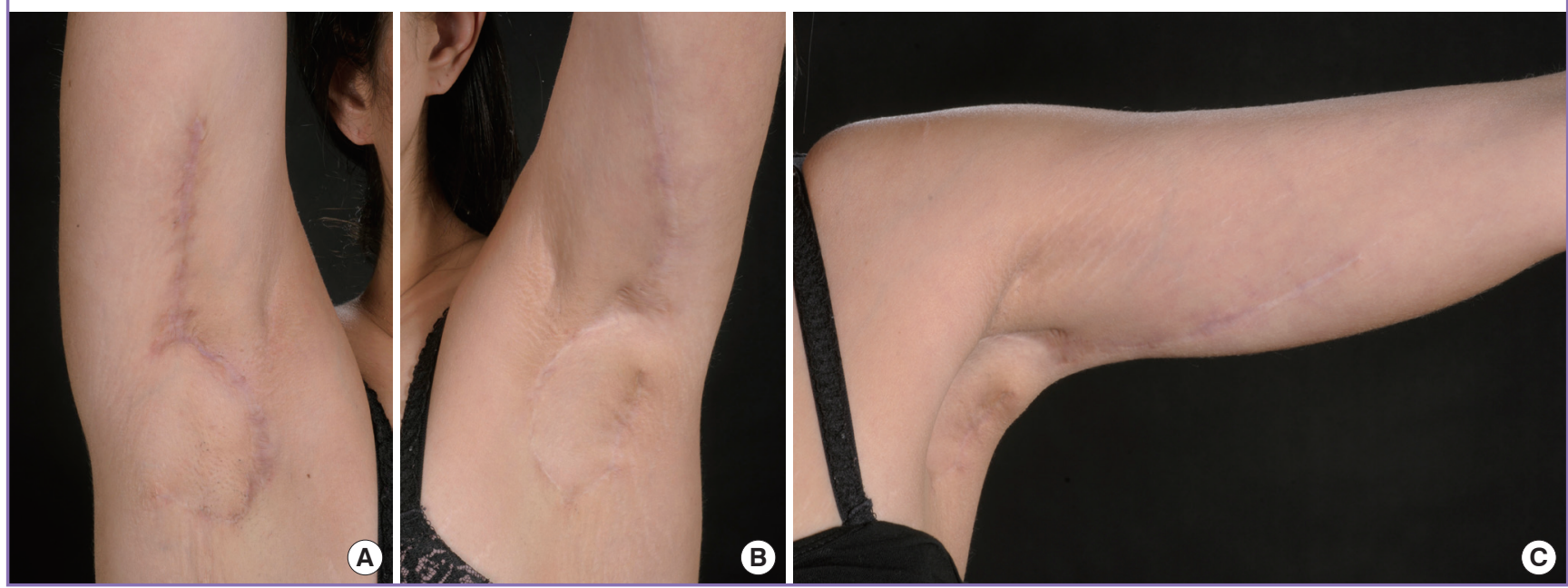

Table 2. Results of study specific questionnaire

\begin{tabular}{|lccc|}
\hline Study specific questionnaire & $\begin{array}{c}\text { Strongly agree } \\
(\%)\end{array}$ & $\begin{array}{c}\text { Agree } \\
(\%)\end{array}$ & $\begin{array}{c}\text { Neutral } \\
(\%)\end{array}$ \\
\hline Quality of life improved after surgery & 50 & 50 & - \\
Felt more confident post-surgery & 50 & 50 & - \\
Satisfied with end result & 75 & - & 25 \\
Will recommend procedure to others & 50 & 25 & 25 \\
\hline
\end{tabular}

summary of the results are shown in Table 2.

\section{DISCUSSION}

The defect left after excision of axillary HS could be closed primarily or left to heal secondarily. Secondary healing is less desirable as it may cause contractures and subsequent limitation in the arm's range of movement leading to stiffening of the shoulders [15]. Commonly cited primary closure methods for axillary HS include the use of direct closure, split thickness skin graft (STSG), fasciocutaneous flap, musculocutaneous flap, parascapular flap, thoracodorsal artery perforator flap (TDAP) and posterior-arm flap $[6,12,16-18]$. A further innovative method for reconstruction has been introduced over the last few years with the addition of the inner-arm flap [19].

Direct closure methods are only possible in limited excision but not in WLE cases $[9,13]$. WLE is defined as $>1 \mathrm{~cm}$ beyond hair bearing skin and has the best cure rate [13]. Hynes et al. [20] demonstrated the use of Topical Negative Pressure dressing in conjunction with STSG and report favourable outcomes with no limitation of arm movement when compared to the contralateral side. Another study demonstrated the use of artifi- cial skin (Terudermis, Terumo Corporation, Tokyo, Japan) whilst awaiting skin granulation after resection in the first stage and the use of STSG for closure in the second stage [21]. The 2 -staged procedure with the use of artificial skin was superior to straight STSG repairs.

The use of the thoracodorsal artery perforator (TDAP) flap was demonstrated in several studies and it was shown able to maintain the diamond shape of the axilla with minimal donor site morbidity [12]. TDAP flaps have also been shown to be superior in postoperative arm abduction compared to other reconstructive procedures [22]. A study with direct comparison of STSG and TDAP flap demonstrated that TDAP flap is superior with reduced recovery time, has less postoperative complications and better quality of life [23]. The posterior-arm flap demonstrated its capabilities of using a similar pedicle for reconstructing axillary as well as thoracic wall defects, but has the downside of leaving a highly visible horizontal scar on the posterolateral aspect of the arm which may not be suitable to certain population groups [18].

There is currently a lack of general consensus on the best reconstructive method [5]. Soldin et al. [13], concluded that different flaps can be used depending on size of the excised defect, recommending a fasciocutaneous flap for small to medium defects and pedicled parascapular flaps for larger defects. It is reasonable to conclude that most studies agree that flap reconstruction is superior to direct closure, STSG, and healing by secondary intention $[8,9,15,22]$.

The flap described in this case series differs from a Hatchet flap since it is a transposition flap with an identified vessel as primary blood supply. It is the elongation of this flap design to re- 
move additional tissue from the inner arm that gives the 'brachioplasty like' improvement to the contour of the arm. This thus turns an otherwise visible donor site scar and more importantly a visible donor site contour abnormality into a longer inner arm scar (with the additional tightening of more of the inner arm tissue) that has benefit to the arm contour. It is designed as a modification of brachioplasty, the tissue that would have been discarded in a brachioplasty is utilized resulting in a postoperative appearance similar to that of a standard brachioplasty.

In our experience, we believe that the branch of the brachial artery utilised in our study has no eponym. A Doppler was not required as the presence of the branch and a pedicle was identified in every case. A study by Hwang et al. [24] which investigated 20 cadaveric upper arms identified 4 constant fasciocutaneous perforators, one in the medial intermuscular septum and three in the lateral intermuscular septum. These perforators were regarded as sufficient and safe for flap design. Our findings are synonymous with cadaveric studies demonstrating highly consistent, reasonable length and diameter inner-arm vessels that can be located with ease $[24,25]$. The lack of an eponymous name for the branch of the brachial artery in itself an area of interest that we hope would raise further discussion and generate future research interest.

With a perforator near the base of the flap, it would be possible for the flap to be islanded. A suprafascial dissection would also be a possibility. The authors have as yet not done this because of the additional blood supply retained by leaving both the fascial base and the skin base of the pedicel intact. In situations where there is no perforator in the base of the flap (type A Lamberty and Cormack) it is anticipated that the flap will still be robust from a vascular perspective because of the inclusion of both suband supra-fascial blood supply.

It would be possible to de-epithelialise the distal portion of the flap if more bulk was needed, but the authors have found this to be unnecessary. This flap is long and narrow. It is likely that the distal most segments will be less well vascularized than the proximal aspect. The distal part that is marked with the crosshatched lines is not for de-epithelialization. It is the part that is discarded. In all cases performed the dog ear always flattened spontaneously with healing and wound resolution. We never had to do a revision.

In all of our cases, the WLE of active disease with an aim to achieve a $0.5-1 \mathrm{~cm}$ margin of normal skin was performed to ensure adequate excision. At all times all active disease including sinuses, abscesses and firm nodules were excised. Usually this represented all hair bearing skin of the axilla. Old healed scars outside this area were not removed unless there was evidence of active disease in the area. We strive to achieve a balance between the creation of an excessively large defect and disease recurrence. There is certainly a possibility in cases presenting with severe, extensive disease involving large surface areas which will not be amenable to the inner-arm flap reconstruction technique. We believe this limitation is not exclusive to our technique, but may also apply to other forms of reconstructive methods. Despite the misleading appearance of residual affected tissue, there has to date (8-36 months follow up) been no disease recurrence or failure of flap closure in any of the patients operated on.

Our study includes all cases performed by one consultant. Taking into consideration the number of consultants and application of the inclusion/exclusion criteria were the reasons for the low number of cases. We acknowledge that 1 out of 5 cases (20\%) had a complication. This in conjunction with the small sample size is a limitation of the study that is worth highlighting. However, it was a minor complication which healed by secondary intention without any major sequelae.

In our study, through the use of the inner-arm transposition flap, we have demonstrated an innovative method for treatment of HS with soft tissue reconstruction. To the authors' knowledge, only one other study has been found in peer reviewed literature on the use of the inner-arm flap for axillary reconstruction. The Alharbi et al. [19] paper had a 25\% complication rate ( 3 out of 12 axilla's had delayed healing) and they experienced widening of scars. In our study we had a similar complication rate of $20 \%$ but did not experience any issues with scar widening postoperative. The lack of details meant that any direct comparison should be performed cautiously. We did however experienced many similarities in that we did not have problems with dog ears, we enjoyed the benefit of a relatively quick dissection, freedom of flap rotation and hidden donor site scarring. We support the conclusion in the Alharbi et al. study that the inner-arm flap is a valuable new tool for axillary reconstruction.

We believe that the effective concealment of all scars under the arms with minimal donor and recipient site scarring in combination with the additional benefit of tightening of the arm tissue in the form of a 'brachioplasty like' effect was favoured by the patient cohort. Although there was one complication, it did not result in flap failure. The inner-arm transposition flap has been shown to provide robust cover to the axillary defect. It is argued to be suitable for most axillary HS cases, regardless of arm circumference. This technique is thought to be reproducible in most settings. Despite the small sample size and in comparison to other reconstructive methods it would be reasonable to conclude the flap is a reliable, effective, and simple method that produces additional benefits. 


\section{ORCID}

Daniel Ching http://orcid.org/0000-0003-3327-2582

\section{REFERENCES}

1. Jemec GB. What's new in hidradenitis suppurativa? J Eur Acad Dermatol Venereol 2000;14:340-1.

2. Jemec GB, Heidenheim M, Nielsen NH. The prevalence of hidradenitis suppurativa and its potential precursor lesions. J Am Acad Dermatol 1996;35:191-4.

3. Slade DE, Powell BW, Mortimer PS. Hidradenitis suppurativa: pathogenesis and management. Br J Plast Surg 2003;56: 451-61.

4. Attanoos RL, Appleton MA, Douglas-Jones AG. The pathogenesis of hidradenitis suppurativa: a closer look at apocrine and apoeccrine glands. Br J Dermatol 1995;133:254-8.

5. Tanaka A, Hatoko M, Tada H, et al. Experience with surgical treatment of hidradenitis suppurativa. Ann Plast Surg 2001;47:636-42.

6. Altmann S, Fansa H, Schneider W. Axillary hidradenitis suppurativa: a further option for surgical treatment. J Cutan Med Surg 2004;8:6-10.

7. Finley EM, Ratz JL. Treatment of hidradenitis suppurativa with carbon dioxide laser excision and second-intention healing.J Am Acad Dermatol 1996;34:465-9.

8. Civelek B, Aksoy K, Bilgen E, et al. Reconstructive options in severe cases of Hidradenitis suppurativa. Cent Eur J Med 2010;5:674-8.

9. Menderes A, Sunay O, Vayvada H, et al. Surgical management of hidradenitis suppurativa. Int J Med Sci 2010;7:2407.

10. Hurley HJ. Axillary hyperhidrosis, apocrine bromhidrosis, hidradenitis suppurativa, and familial benign pemphigus: surgical approach. In: Roenigk RK, Roenigk HH, editors. Dermatologic surgery. New York: Marcel Dekker; 1989. p.729-39.

11. Giovannini UM, Giannasi S. Fasciocutaneous flap to treat verneuil disease (hidradenitis suppurativa) in the axillary region. Ann Plast Surg 2003;50:108-9.

12. Ortiz CL, Castillo VL, Pilarte FS, et al. Experience using the thoracodorsal artery perforator flap in axillary hidradentitis suppurativa cases. Aesthetic Plast Surg 2010;34:785-92.

13. Soldin MG, Tulley P, Kaplan H, et al. Chronic axillary hi- dradenitis: the efficacy of wide excision and flap coverage. Br J Plast Surg 2000;53:434-6.

14. Carr T, Moss T, Harris D. The DAS24: a short form of the Derriford Appearance Scale DAS59 to measure individual responses to living with problems of appearance. $\mathrm{Br} \mathrm{J}$ Health Psychol 2005;10:285-98.

15. Rehman N, Kannan RY, Hassan S, et al. Thoracodorsal artery perforator (TAP) type I V-Y advancement flap in axillary hidradenitis suppurativa. Br J Plast Surg 2005;58:441-4.

16. Buyukasik O, Osmanoglu CG, Polat Y, et al. A life-threatening multilocalized hidradenitis suppurativa case. MedGenMed 2005;7:19.

17. Geh JL, Niranjan NS. Perforator-based fasciocutaneous island flaps for the reconstruction of axillary defects following excision of hidradenitis suppurativa. Br J Plast Surg 2002;55: 124-8.

18. Schmidt M, Dunst-Huemer KM, Lazzeri D, et al. The versatility of the islanded posterior arm flap for regional reconstruction around the axilla. J Plast Reconstr Aesthet Surg 2015;68:953-9.

19. Alharbi M, Perignon D, Assaf N, et al. Application of the inner arm perforator flap in the management of axillary hidradenitis suppurativa. Ann Chir Plast Esthet 2014;59:29-34.

20. Hynes PJ, Earley MJ, Lawlor D. Split-thickness skin grafts and negative-pressure dressings in the treatment of axillary hidradenitis suppurativa. Br J Plast Surg 2002;55:507-9.

21. Iida N, Fukushima K, Kanzaki A. A two-stage technique using a bovine dermal substitute to treat axillary hidradenitis. European Journal of Plastic Surgery 2005;28:359-63.

22. Busnardo FF, Coltro PS, Olivan MV, et al. The thoracodorsal artery perforator flap in the treatment of axillary hidradenitis suppurativa: effect on preservation of arm abduction. Plast Reconstr Surg 2011;128:949-53.

23. Wormald JC, Balzano A, Clibbon JJ, et al. Surgical treatment of severe hidradenitis suppurativa of the axilla: thoracodorsal artery perforator (TDAP) flap versus split skin graft. J Plast Reconstr Aesthet Surg 2014;67:1118-24.

24. Hwang K, Lee WJ, Jung CY, et al. Cutaneous perforators of the upper arm and clinical applications. J Reconstr Microsurg 2005;21:463-9.

25. Perignon D, Havet E, Sinna R. Perforator arteries of the medial upper arm: anatomical basis of a new flap donor site. Surg Radiol Anat 2013;35:39-48. 